\title{
Monitorização da oferta do oxigênio suplementar em neonatos: desafios e potências*
}

\author{
Monitoring of supplemental oxygen supply in neonates: challenges and potentialities
}

Vanessa Oliveira Ossola da Cruz ${ }^{1}$, Luciana da Silva Lanzillotti ${ }^{2}$, Andrea $\mathrm{Zin}^{2}$, Aline Piovezan Entringer ${ }^{2}$, Marcelle Campos Araujo ${ }^{2}$, Roberto Carlos Lyra da Silva ${ }^{1}$

Objetivo: avaliar as práticas da equipe multidisciplinar na monitorização de oxigênio suplementar ofertado para recém-nascidos. Métodos: estudo transversal, com dados provenientes de um instrumento observacional relacionado à monitorização da oferta do oxigênio. A coleta de dados foi em um mês, no plantão diurno, o qual foi observado a prática de 104 profissionais resultando em 22 dias observados e 188 monitores checados pela própria pesquisadora. Resultados: a relação de monitores corretos, comparado a configuração está desligado e alterado o ajuste, resultou em mediana de 0,43 e desvio padrão de 0,17 . Ou seja, 57,0\% dos monitores não estavam no ajuste adequado. Vale destacar que $100,0 \%$ dos recém-nascidos estavam monitorizados e em uso do blender. Conclusão: constata-se que as práticas desenvolvidas pela equipe multidisciplinar nesta instituição estão de acordo com a Sociedade Brasileira de Pediatria diante de todos os recém-nascidos estarem monitorizados e em uso do blender na oferta do oxigênio suplementar.

Descritores: Oxigenoterapia; Recém-Nascido; Enfermagem; Neonatologia; Equipe Interdisciplinar de Saúde.

Objective: to evaluate the practices of the multidisciplinary team in monitoring supplemental oxygen offered to newborns. Methods: cross-sectional study with data from an observational instrument related to oxygen supply monitoring. Data collection occurred within one month, in the morning shift, and involved the observation of the practice of 104 professionals during a total of 22 days, and the checking of 188 monitors by the researcher. Results: the ratio monitors in the correct setting/monitors turned off or with altered setting resulted in a median of 0.43 and a standard deviation of 0.17 . That is, $57.0 \%$ of the monitors were not properly set up. It was noteworthy that monitoring and blender were used in $100.0 \%$ of the newborns. Conclusion: it was found that the practices developed by the multidisciplinary team in this institution are in agreement with the Brazilian Society of Pediatrics, as monitoring and blender were used in all newborns receiving supplemental oxygen.

Descriptors: Oxygen Inhalation Therapy; Infant, Newborn; Nursing; Neonatology; Patient Care Team.

\footnotetext{
*Extraído do trabalho de conclusão de curso "Avaliação das boas práticas na monitorização da oferta de oxigênio suplementar em uma Unidade de Terapia Intensiva Neonatal”, Instituto Fernandes Figueira, 2017.

${ }^{1}$ Universidade Federal do Estado do Rio de Janeiro. Rio de Janeiro, RJ, Brasil.

${ }^{2}$ Instituto Fernandes Figueira. Rio de Janeiro, RJ, Brasil.
} 


\section{Introdução}

Nos últimos anos, o uso do oxigênio suplementar em recém-nascidos pré-termos, tem sido uma preocupação dos profissionais de saúde. A oxigenoterapia suplementar é uma das intervenções terapêuticas mais utilizada na Unidade de Terapia Intensiva Neonatal. Desse modo, pode-se afirmar que, da mesma forma que a comida, o calor, e a água, também o oxigênio é vital para manter a vida, no entanto, seu excesso pode causar danos ${ }^{(1)}$.

A partir de 1940, o uso liberal do oxigênio em bebês pré-termos resultou no aumento de casos de Retinopatia da Prematuridade. Na década de 19601970, coube a restrição de seu uso, havendo redução na incidência da Retinopatia da Prematuridade, aumentando, entretanto, a hipóxia e a mortalidade. Cabe destacar que, tanto a hiperóxia quanto a hipóxia, estão associadas a morbidades como displasia broncopulmonar, lesão cerebral e a Retinopatia da Prematurida$\mathrm{de}^{(2-6)}$.

Uma das medidas para minimizar as ocorrências dessas morbidades, é a monitorização da saturação de oxigênio através do uso de oxímetro de pulso, que tem como objetivo, manter os níveis de oxigênio dentro de limites seguros, permitindo o controle mais preciso da oferta de uma fração de oxigênio inspirado de acordo com as necessidades do paciente, através do respirador ${ }^{(7-8)}$.

No meio científico ainda há incertezas de qual é o melhor parâmetro para o alarme no monitor, e assim determinar o limite de saturação de oxigênio ao recém-nascido. No entanto, há um estudo que recomenda o ajuste do alarme entre 89-95,0\% para obter a saturação de oxigênio entre $90-94,0 \%$ ao recém-nascido. Pois reduz a hipóxia, hiperóxia, volutrauma, as morbidades associadas e a mortalidade ${ }^{(9)}$.

A administração de oxigênio suplementar em recém-nascido, é um desafio que necessita de atuação multidisciplinar entre a equipe médica, de enfermagem e de fisioterapia respiratória, para minimizar as complicações associadas à hipóxia e hiperóxia, pro- porcionando um cuidado singular, qualificado e humanizado. Dessa forma, o objetivo desse estudo foi avaliar as práticas da equipe multidisciplinar na monitorização de oxigênio suplementar ofertado para recém-nascidos.

\section{Métodos}

O delineamento do estudo é transversal e foi desenvolvido em uma Unidade de Terapia Intensiva Neonatal com capacidade para 14 leitos na cidade do Rio de Janeiro, Brasil.

Nessa unidade há muitos recém-nascidos graves e crônicos, além de ser uma instituição de ensino (graduandos, mestrandos, doutorandos e residentes) e de referência na saúde da mulher, da criança e do adolescente. Assim sendo, para se obter o maior quantitativo de profissionais na unidade, não atrapalhar a dinâmica do setor por ser altamente corrido e agitado e coletar os dados da observação participante de forma que os profissionais não percebessem, para não interferir nos resultados, o grupo de pesquisa decidiu ser em um mês, no plantão diurno, 07h às $19 \mathrm{hrs}$, de segunda a sexta-feira, a partir da construção de um instrumento observacional no período de novembro de 2016, o qual resultou em 22 dias observados pela pesquisadora responsável pelo estudo.

Os participantes da pesquisa observados foram 104 profissionais, os quais foram informados sobre a pesquisa e seu método, sendo: 11 médicos neonatologistas (plantonistas e rotinas staffs), 13 médicos residentes (residente de terceiro ano e residente de quarto ano), 2 fisioterapeutas plantonistas, 2 fisioterapeutas residentes, 20 enfermeiros (rotina e plantonistas), 11 enfermeiros residentes (residentes de primeiro ano e residente de segundo ano) e 45 técnicos de enfermagem que atuam na unidade.

Para avaliar as boas práticas na monitorização da oferta do oxigênio suplementar ao recém-nascido foram baseadas nas diretrizes da Sociedade Brasileira de Pediatria ${ }^{(10-11)}$ obtendo 100,0\% das observações dessas práticas, que são: usar o oxímetro de pulso, não 
passar valores acima de 95,0\% no ajuste de saturação de oxigênio ao recém-nascido e utilizar o blender (mistura oxigênio e ar comprimido).

Na produção dos dados acerca do cenário da pesquisa, as seguintes informações foram extraídas do instrumento observacional, resultando nas seguintes variáveis: ocupação dos leitos, monitorização do oxigênio (os recém-nascidos estavam em uso do oxímetro de pulso), observação da prática dos profissionais frente à configuração do monitor (pesquisador verifica os monitores e qual ajuste está programado ao recém-nascido em oferta de oxigênio suplementar), quais os métodos de oferta de oxigênio encontrados na unidade e qual a relação profissional/recém-nascido.

É importante destacar que os recém-nascidos não constituem objeto de pesquisa, e sim as práticas desenvolvidas pelos profissionais na monitorização da oferta do oxigênio suplementar. Como critério de exclusão, teve-se os monitores dos recém-nascidos cardiopatas, pois utiliza-se parâmetros diferentes, sendo dispensado a observação destes nesse estudo.

Os dados foram organizados com auxílio dos programas Microsoft Excel® e Microsoft Word®, analisados a partir da estatística descritiva, utilizando as medidas de tendência central e dispersão. 0 protocolo de pesquisa foi aprovado pelo Comitê de Ética em Pesquisa do Instituto Fernandes Figueira, com o parecer $n^{\circ} 1.894 .151 / 2016$.

\section{Resultados}

Dos 104 profissionais de saúde observados obteve-se a mediana de 10 anos de formado, três anos do tempo de atuação na unidade e seis anos de atuação na neonatologia. 0 perfil é 37 (35,6\%) têm especialização lato sensu, 76 (73,1\%) são da equipe de enfermagem, 54 (51,9\%) compõe o quadro permanente da instituição, 83 (79,8\%) são plantonistas, a carga horária semanal: 75 (72,1\%) são 40 hrs e 57 $(54,8 \%)$ não trabalha em outra instituição (Tabela 1$)$.
Tabela 1 - Perfil dos profissionais observados

\begin{tabular}{|c|c|}
\hline Variáveis & n (\%) \\
\hline \multicolumn{2}{|l|}{ Escolaridade } \\
\hline Técnico & $34(32,7)$ \\
\hline Graduado & $16(15,4)$ \\
\hline Especialização lato sensu & $37(35,6)$ \\
\hline Mestrado & $13(12,5)$ \\
\hline Doutorado & $3(2,9)$ \\
\hline $\mathrm{PhD}$ & $1(1,0)$ \\
\hline \multicolumn{2}{|l|}{ Cargo } \\
\hline Enfermagem & $76(73,1)$ \\
\hline Médico & $24(23,1)$ \\
\hline Fisioterapia & $4(3,8)$ \\
\hline \multicolumn{2}{|l|}{ Vínculo empregatício } \\
\hline Quadro permanente & $54(51,9)$ \\
\hline Bolsa de residência & $26(25,0)$ \\
\hline Contrato de trabalho & $23(22,1)$ \\
\hline Pesquisador - Mestrado & $1(1,0)$ \\
\hline \multicolumn{2}{|c|}{ Função que exerce no momento } \\
\hline Plantonista & $83(79,8)$ \\
\hline Residente & $15(14,4)$ \\
\hline Rotina/diarista & $6(5,8)$ \\
\hline \multicolumn{2}{|l|}{ Carga horária seminal (horas) } \\
\hline 20 & $1(1,0)$ \\
\hline 30 & $2(1,9)$ \\
\hline 40 & $75(72,1)$ \\
\hline$\geq 60$ & $26(25,0)$ \\
\hline \multicolumn{2}{|l|}{ Trabalha em outra instituição } \\
\hline Não & $57(54,8)$ \\
\hline Sim & $47(45,2)$ \\
\hline
\end{tabular}

A média/mediana de paciente-dia foi de 15 bebês, com o máximo de 18 bebês (ocupação 129,0\%) e mínimo de 12 (ocupação 86,0\%). 0 desvio padrão foi 1,69 e o intervalo interquartil foi 14;15;16.

A média de recém-nascidos em uso de algum método de oferta de oxigênio suplementar foi de 8,4; o desvio padrão foi 1; a mediana foi de 9 e intervalo interquartil foi 8;9;9. A média de pacientes em uso da ventilação mecânica foi 4,32; o desvio padrão de 1,67; a mediana de 5 e o intervalo interquartil foi 2,$25 ; 5 ; 6$. A média de pacientes utilizando pressão positiva contí- 
nua nas vias aéreas foi de 3,5; o desvio padrão de 1,18; a mediana de 3 e o intervalo interquartil foi de $3 ; 3 ; 4$. E a média de pacientes em uso de cateter nasal foi de 0,5 ; o desvio padrão de 1 ; mediana de 0,5 e o intervalo interquartil de $0 ; 0,5 ; 1$. Cabe destacar que $100,0 \%$ dos recém-nascidos estavam monitorizados e utilizavam-se o blender.

A média diária de monitores checados foi 8,54; o desvio padrão foi 1,1; a mediana diária foi de 9 e o intervalo interquartil foi de 8;9;9. A média diária de monitores desligados foi de 4,41; o desvio padrão de 1,74; a mediana diária de 4 e o intervalo interquartil de 3;4;5,75. A média diária de monitores no ajuste correto foi de 3,5; o desvio padrão de 1,5; a mediana diária foi de 4 e o intervalo interquartil de 3;4;4,75. E a média diária de monitores no ajuste alterado foi de 0,64 ; o desvio padrão de 0,73 ; a mediana diária foi de 0,5 e o intervalo interquartil de 0;0,5;1. Destaca-se que nos três primeiros dias de coleta de dados a pesquisadora encontrou um recém-nascido com dois monitores, pré e pós ductal, diante da sua gravidade. A partir do quarto dia, após estabilização, o bebê ficou com apenas um monitor.

Quanto à relação de monitores no ajuste correto, comparado a estar desligado ou alterado o ajuste, resultou em uma média de 0,41 ; o desvio padrão de 0,17 ; a mediana de 0,43 e o intervalo interquartil de 0,$31 ; 0,43 ; 0,54$. Ou seja, em torno de $57,0 \%$ dos monitores não estavam no ajuste adequado. Assim a recomendação, não passar valores acima de 95,0\% no ajuste de saturação de oxigênio ao recém-nascido não foi contemplado.

Após a observação, quando a pesquisadora obtinha a configuração do ajuste do alarme desligado, ou o valor diferente do preconizado, em postura ética, ia ao encontro dos profissionais que estavam responsáveis pelos cuidados do recém-nascido a fim de orientá-los, obtendo sucesso ao conseguir que eles configurassem o monitor conforme o parâmetro institucional (alarme no monitor valor entre 89 - 95,0\%) (Tabela 2).
Tabela 2 - Distribuição da configuração do monitor

\begin{tabular}{|c|c|c|c|c|}
\hline Dias & Correto & Desligado & Alterado & Total \\
\hline 1 & 0 & 8 & 1 & 9 \\
\hline 2 & 3 & 7 & 0 & 10 \\
\hline 3 & 3 & 7 & 0 & 10 \\
\hline 4 & 5 & 4 & 0 & 9 \\
\hline 5 & 1 & 7 & 1 & 9 \\
\hline 6 & 2 & 6 & 1 & 9 \\
\hline 7 & 4 & 5 & 1 & 10 \\
\hline 8 & 4 & 5 & 0 & 9 \\
\hline 9 & 3 & 6 & 0 & 9 \\
\hline 10 & 4 & 5 & 0 & 9 \\
\hline 11 & 5 & 4 & 1 & 10 \\
\hline 12 & 4 & 4 & 0 & 8 \\
\hline 13 & 5 & 3 & 0 & 8 \\
\hline 14 & 5 & 3 & 0 & 8 \\
\hline 15 & 6 & 3 & 0 & 9 \\
\hline 16 & 5 & 2 & 2 & 9 \\
\hline 17 & 4 & 3 & 0 & 7 \\
\hline 18 & 3 & 3 & 2 & 8 \\
\hline 19 & 1 & 3 & 2 & 6 \\
\hline 20 & 3 & 3 & 1 & 7 \\
\hline 21 & 3 & 3 & 1 & 7 \\
\hline 22 & 4 & 3 & 1 & 8 \\
\hline
\end{tabular}

O dimensionamento diário-profissional para a equipe de enfermagem foi a média/mediana de 2 enfermeiros staffs (desvio padrão de 0,2 ; intervalo interquartil de 2;2;2), a média de 2,7 e a mediana de 3 enfermeiros residentes (desvio padrão de 1,13; intervalo interquartil de 2,25;3;3) e a média de 5,95 e a mediana de 6 técnicos de enfermagem (desvio padrão de 1,17; intervalo interquartil de 5,25;6;7) para uma média/mediana de 15 leitos ocupados (desvio padrão de 1,7; intervalo interquartil de 14;15;16).

Para a equipe médica foi a média 2,27 e a mediana de 2 médicos staffs de neonatologia (desvio padrão de 0,88 ; intervalo interquartil de 2;2;3) e a média de 6,72 e a mediana de 7 residentes médicos de neonatologia (desvio padrão de 1,72; intervalo interquartil de 6;7;7,75) para uma média/mediana de 15 leitos ocupados.

E para a equipe de fisioterapia foi a média de 0,6 e a mediana de 0 fisioterapeuta staff (desvio pa- 
drão de 0,73 ; intervalo interquartil de $0 ; 0 ; 1$ ) e a média de 0,2 e a mediana de 0 residente de fisioterapia para uma média de 8,4 e uma mediana de 9 recém-nascidos em oxigenoterapia (desvio padrão de 1; intervalo interquartil de 8;9;9). Dos 22 dias observados, há 12 $(55,0 \%)$ dias sem fisioterapeuta staff. 0 funcionamento da fisioterapia no setor é de segunda à sexta-feira em torno das 8-14 horas, exceto feriados.

Ademais avalia-se que a equipe multiprofissional desenvolveu as boas práticas ao recém-nascido em oxigenoterapia, pois todos estavam monitorizados e em uso do blender. No entanto, o ajuste correto no monitor não foi contemplado em sua maioria. Tal resultado pode ter relação por excesso de trabalho, pois houve a capacidade excedida e com dimensionamento profissional inadequado; e pode haver o desconhecimento da equipe da importância do ajuste correto e suas repercussões a saúde do recém-nascido.

\section{Discussão}

Pode-se dizer que o estudo é limitado no tempo de observação na unidade, pois ocorreu apenas de segunda à sexta-feira, no período diurno, em apenas um mês. Além disso, tem-se o fato de que a instituição por ser referência, pode levar a dificuldade de comparar com outras unidades de terapia intensiva neonatal.

Este estudo possibilitou observar que todos os recém-nascidos estão monitorizados, isso é uma boa prática profissional e contribui para a prática clínica e para a sociedade, pois gera a cultura de segurança ${ }^{(12-13)}$ aos bebês, trazendo sentimentos impactantes de positividade aos pais, isto é, eles se sentem bem mais confiantes em relação à segurança de seus bebês.

Ademais, constata-se que a demanda de cuidados é elevada, tanto para a oxigenoterapia, quanto para o quantitativo de recém-nascidos na unidade, que pode ter contribuído para a diminuição de monitores ajustados corretamente. Assim, como contribuição para a prática clínica e para a sociedade, torna-se imprescindível que tais problemáticas apontadas, sejam discutidas a nível gerencial, e com a equipe res- ponsável pela educação permanente, pois são fatores importantíssimos, que se não forem solucionados, podem acarretar grandes problemas no futuro para saúde, comprometendo vidas e implicando na saúde pública neonatal ${ }^{(14-15)}$.

Outro fator importante é a ventilação mecânica, que tem o objetivo de fornecer trocas gasosas "aceitáveis" a fim de minimizar distúrbios circulatórios e lesão pulmonar. Em nosso estudo obtivemos mais casos em uso de ventilação mecânica (mediana de 5 casos-dia). Logo, as boas práticas ofertadas pela equipe multidisciplinar a esse recém-nascido através da monitorização do oxigênio nessa modalidade ventilatória minimizam a hipóxia e a hiperóxia. Pois a ventilação mecânica tem o princípio de recuperar o pulmão com atelectasia insuflando-o e otimizando o volume pulmonar prevenindo o colabamento dos alvéolos com o uso mínimo do oxigênio ${ }^{(9)}$.

Em outro estudo, 43 bebês que necessitaram de oxigênio suplementar, por meio de suporte respiratório não invasivo, utilizando três diferentes faixas alvo de saturação de oxigênio com um ponto médio fixo de 90,0\%: 1(86-94,0\%); 2 (88-92,0\%); e 3 (89-91,0\%), obteve o tempo gasto em hipoxemia graves (saturação de oxigênio $<80,0 \%$ ), foi significativamente reduzida durante os intervalos-alvo reduzidos de fração de oxigênio inspirado (88-92,0\%; 1,9\%, 89-91,0\%; 1,7\%) em comparação com o amplo valor alvo (86-94,0\%; $3,4 \%, p<0,001)$. Além disso, os profissionais acabam tolerando a hiperóxia para evitar crises de hipóxia. Logo, recomendam a mediana desejada de $\pm 2,0 \%$ na redução do tempo gasto em hipoxemia, sem aumentar o risco de hiperoxemia ${ }^{(7)}$.

Constata-se que o artigo traz diferentes valores no ajuste de saturação de oxigênio. Pois ainda se discute os valores de referências cabendo mais estudos dentro dessa temática. Assim, colaborando com o nosso trabalho, dois artigos trazem a faixa alvo estreita ótima de saturação de oxigênio de $90-94,0 \%{ }^{(2,9)}$ e um para o limite inferior de $89,0 \%$ no ajuste do monitor $^{(9,14)}$. Tais conhecimentos são imprescindíveis na atuação da equipe multiprofissional para o adequado 
manejo do ajuste de saturação de oxigênio. Pois implica na melhora clínica e na minimização da morbimortalidade neonatal relacionada a oxigenoterapia.

A configuração do monitor exige a atuação ativa dos profissionais de saúde, pois ao verificarem e ajustarem conforme a recomendação institucional estão contribuindo diretamente na qualidade da assistência ao recém-nascido na oferta de oxigênio suplementar, e minimizando as morbidades relacionadas ao excesso de oxigênio como a retinopatia da prematuridade ${ }^{(9,15)}$.

0 estudo realizado em uma Unidade de Terapia Intensiva Neonatal obteve o ajuste do monitor no modo silencioso em todos os dias da realização da pesquisa. Tal atitude expõe o recém-nascido aos riscos no uso do oxigênio suplementar, pois não há a sinalização do alarme do monitor, podendo haver uma hipóxia, hiperóxia e interferir na melhora clínica desse recém-nascido. Assim desperta-nos para o questionamento se os profissionais que atuam nessa unidade têm o conhecimento dos valores para o ajuste do alarme e se sabem quais os cuidados ofertados ao recém-nascido em oxigenoterapia ${ }^{(15)}$.

Outro ponto vital, que precisa ser visto, é a capacidade excedida na unidade. Pois observou-se que, mantém-se o quantitativo de profissionais cuidando do recém-nascido, no entanto, a qualidade da assistência pode ser prejudicada, devido a demanda dos bebês que necessitam de atenção, e estão em maior número comparado ao número que a equipe/estrutura está preparada a atender(15).

O trabalho desenvolvido em uma Unidade de Terapia Intensiva Neonatal verificou que a cada cinco períodos observados, um estava com superlotação. Portanto, obteve-se uma demanda superior à sua capacidade de ocupação, o que pode induzir a ocorrência de altas precoces, e acarretar cuidados na Unidade de Cuidados Intermediários de um nível de maior complexidade que o recomendado para essa unidade, evidenciando a necessidade de um quadro ainda maior de profissionais de enfermagem, principalmente enfermeiros, para o monitoramento dos bebês, também nesse setor ${ }^{(15)}$.
Com intuito de reduzir a hipóxia ${ }^{(16)}$ e a hiperó$x i^{(17)}$, faz-se necessário um trabalho multidisciplinar. Ou seja, a equipe médica, a fisioterapia e a equipe de enfermagem trabalhando juntos, num trabalho complementar, desempenhando um papel fundamental na gestão das metas de saturação de oxigênio. Além do desenvolvimento de diretrizes e políticas baseadas em evidências para promover uma abordagem segura e consistente nos cuidados prestados por todos os membros da equipe da Unidade de Terapia Intensiva Neonatal ${ }^{(15,18)}$.

Quanto ao dimensionamento do pessoal, toda a equipe profissional em Unidade de Terapia Intensiva Neonatal tipo III tem à proporção de um profissional para 1,33 paciente nos diferentes turnos de trabalho, diante da distribuição percentual de 52,0\% de enfermeiro e $48,0 \%$ de técnico. A portaria do Ministério da Saúde preconiza: um enfermeiro plantonista por turno para cinco leitos, e um técnico de enfermagem para dois leitos por turno. Aos médicos, um médico plantonista para cada 10 leitos em cada turno. E à fisioterapia, um fisioterapeuta exclusivo para cada 10 leitos em cada turno ${ }^{(19)}$. Dessa forma, aplicando tais achados sob os profissionais staff obteve-se a não conformidade na relação profissional/paciente.

Pesquisadores destacam que o desenvolvimento de pesquisas nessa temática é pouco discutida e abordada, incluindo unidades intensivas e neonatais ${ }^{(7,15)}$. Logo é importante o desenvolvimento de protocolos operacionais padrões com o treinamento de toda a equipe multidisciplinar, pensando em sua pronta resposta aos alarmes, os ajustes dos monitores e sensibilizando-os sobre os riscos da hipóxia e hiperóxia para a vida do recém-nascido em oxigenoterapia $^{(15)}$.

\section{Conclusão}

Ao longo do estudo, constatou-se que as práticas desenvolvidas pela equipe multidisciplinar nesta instituição estão de acordo com a Sociedade Brasileira de Pediatria diante de todos os recém-nascidos esta- 
rem monitorizados e em uso do blender na oferta do oxigênio suplementar. No entanto, o critério não passar valores acima de 95,0\% no ajuste de saturação de oxigênio ao recém-nascido não foi contemplado, pois obteve-se menos de 50,0\% dos monitores configurados corretamente.

\section{Colaborações}

Cruz VO0, Lanzillotti LS, Zin A, Entringer AP e Araujo MC contribuíram na concepção e projeto, análise e interpretação dos dados, redação do artigo, revisão crítica relevante do conteúdo intelectual e aprovação final da versão a ser publicada. Silva RCL realizou a revisão crítica relevante do conteúdo intelectual e aprovação final da versão a ser publicada.

\section{Referências}

1. Cummings JJ, Lakshminrusimha S. Oxygen saturation targeting by pulse oximetry (SpO2) in the Extremely Low Gestational Age Neonate (ELGAN): a quixotic quest. Curr Opin Pediatr. 2017; 29(2):153-8. doi: http://dx.doi.org/10.1097/ MOP.0000000000000458

2. Zoban P. Optimal oxygen saturation in extremely premature neonates. Physiol Res. 2019; 68(2):171-8. doi: http://dx.doi.org/10.33549/ physiolres. 933987

3. Cummings JJ, Polin RA. Oxygen targeting in extremely low birth weight infants. Pediatrics. 2016; 138(2):e20161576. doi: http://dx.doi. org/10.1542/peds.2016-1576

4. Van Zanten HA, Kuypers KLAM, Stenson BJ, Bachman TE, Pauws SC, Te PAB. The effect of implementing an automated oxygen control on oxygen saturation in preterm infants. Arch Dis Child Fetal Neonatal Ed. 2017; 102(5):395-9. doi: http://dx. doi.org/10.1136/archdischild-2016-312172

5. Torres-Cuevas I, Parra-Llorca A, Sánchez-Illana A, Nuñez-Ramiro A, Kuligowski J, Cháfer-Pericás C, et al. Oxygen and oxidative stress in the perinatal period. Redox Biol. 2017; 12:674-81. doi: http:// dx.doi.org/10.1016/j.redox.2017.03.011
6. Manley BJ, Kuschel CA, Elder JE, Doyle LW, Davis PG. Higher rates of retinopathy of prematurity after increasing oxygen saturation targets for very preterm infants: experience in a single center. J Pediatr. 2016; 168:242-4. doi: http://dx.doi. org/10.1016/j.jpeds.2015.10.005

7. Van den Heuvel MEN, Van Zanten HA, Bachman TE, Te PAB, Van Kaam AH, Onland W. Optimal target range of closed-loop inspired oxygen support in preterm infants: a randomized cross-over study. J Pediatr. 2018; 197:36-41. doi: http://dx.doi. org/10.1016/j.jpeds.2018.01.077

8. Dargaville PA, Sadeghi Fathabadi O, Plottier GK, Lim K, Wheeler KI, Jayakar R, et al. Development and preclinical testing of an adaptive algorithm for automated control of inspired oxygen in the preterm infant. Arch Dis Child Fetal Neonatal Ed. 2017; 102(1):31-6. doi: http://dx.doi. org/10.1136/archdischild-2016-310650

9. Sweet DG, Carnielli V, Greisen G, Hallman M, Ozek E, Plavka R, et al. European consensus guidelines on the management of respiratory distress syndrome - 2016 Update. Neonatology. 2017; 111(2):10725. doi: http://dx.doi.org/10.1159/000448985

10. Almeida MFB, Guinsburg R. Reanimação do recém-nascido $\geq 34$ semanas em sala de parto: Diretrizes 2016 da Sociedade Brasileira de Pediatria [Internet]. 2016 [citado 2019 Abr 12]. Disponível em:https://www.sbp.com.br/fileadmin/user_ upload/sSBPReanimacaoRNMaior34semanas26jan2016.pdf

11. Guinsburg R, Almeida MFB. Reanimação do recém-nascido < 34 semanas em sala de parto: Diretrizes 2016 da Sociedade Brasileira de Pediatria [Internet]. 2016 [citado 2019 Abr 12]. Disponível em:https://www.sbp.com.br/fileadmin/user_ upload/nimacaoPrematuroMenor34semanas26jan2016.pdf

12. Tomazoni A, Rocha PK, Kusahara DM, Souza AIJ, Macedo TR. Evaluation of the safety culture of the patient in neonatology units from the perspective of the multiprofessional team. Texto Contexto Enferm. 2015; 24(1):161-9. doi: http://dx.doi. org/10.1590/0104-07072015000490014

13. Panagos PG, Pearlman SA. Creating a Highly Reliable Neonatal Intensive Care Unit Through Safer Systems of Care. Clin Perinatol. 2017; 44(3):64562. doi: dx.doi.org/10.1016/j.clp.2017.05.006 
14. Manja V, Saugstad OD, Lakshminrusimha S. Oxygen saturation targets in preterm infants and outcomes at 18-24 months: a systematic review. Pediatrics. 2017; 39(1):e20161609. doi: http:// dx.doi.org/10.1542/peds.2016-1609

15. Marta CB, Junior HCS, Costa DJ, Martins GM, Silva $\mathrm{RC}$, Pereira LS. The nursing team before alarm triggering in the neonatal intensive care unit. Rev Pesqui Cuid Fundam Online. 2016; 8(3):4773-9. doi: http://dx.doi.org/10.9789/2175-5361.2016. v8i3.4773-4779

16. Badurdeen S, Roberts C, Blank D, Miller S, Stojanovska V, Davis P, et al. Haemodynamic instability and brain injury in neonates exposed to hypoxia-ischaemia. Brain Sci. 2019; 9(3):49. doi: http://dx.doi.org/10.3390/brainsci9030049
17. Stenson BJ. Oxygen saturation targets for extremely preterm infants after the NeOProM trials. Neonatology. 2016; 109(4):352-8. doi: http://dx.doi.org/10.1159/000444913.

18. Paul M. Oxygen administration to preterm neonates in the delivery room: Minimizing oxidative stress. Adv Neonatal Care. 2015; 15(2):94-103. doi: http://dx.doi.org/10.1097/ ANC.0000000000000147

19. Luna A, Branco L, Beleza L. Nursing workload in neonatal ICU: application of the nursing activities score tool. Rev Pesqui Cuid Fundam Online. 2017; 9(1):144-51. doi: http://dx.doi. org/10.9789/2175-5361.2017.v9i1.144-151 